\title{
Radial mean velocity in the solar neighbourhood
}

\author{
R. Cubarsi and S. Alcobé \\ Dept. Matemàtica Aplicada IV, Universitat Politècnica de Catalunya, 08034 Barcelona, Catalonia, Spain \\ e-mail: rcubarsi@mat.upc.es
}

Received 19 May 2006 / Accepted 22 June 2006

\begin{abstract}
A large sample with the full space motions drawn from the Hipparcos Catalogue allows us to select some nested subsamples containing an increasing number of thick disk stars. Segregated thick disk populations show a progressive decreasing vertex deviation according to a trend of axial symmetry, as well as an increasing velocity dispersion and mean age, which are consistent with a trend of steady state. Thus, we propose to extrapolate the oldest thick disk by using a quite general kinematic model, that is time dependent and with point-to-point axial symmetry, until reaching a hypothetical stellar population with axial symmetry, vanishing radial differential movement, and steady state. In the Galactic plane, the extrapolated population determines the point of the velocity space with differential rotation alone, that is with no net radial motion. The present work improves the estimation of the local radial mean velocity that we obtained in a previous work, where a raw linear extrapolation was performed. Now we complete the analysis by using a more precise estimation based on the kinematic model. The resulting no net radial motion point has radial heliocentric velocity $U=-20 \pm 1 \mathrm{~km} \mathrm{~s}^{-1}$ and rotation heliocentric velocity $V=-82 \pm 2 \mathrm{~km} \mathrm{~s}^{-1}$. During the disk heating process, the disk populations lose angular momentum and radial motion while increasing the velocity dispersion. And, in particular, the thick disk loses its total radial motion before reaching the steady state, in an attempt to recover axiality. The new estimation stresses previous conclusions about the vanishing radial mean velocity of the stars associated with the Hyades-Pleiades supercluster, originated by large molecular clouds.
\end{abstract}

Key words. stars: kinematics - stars: Population II - Galaxy: kinematics and dynamics

\section{Introduction}

The purpose of the present work is to improve a previous study (Alcobé \& Cubarsi 2005) in which, as a consequence of the kinematic analysis of disk populations in the solar neighbourhood, the parameters of thin and thick disk components were obtained according to nearly ellipsoidal velocity distributions. As the whole sample was enlarged, by selecting stars with greater heliocentric velocity, and after extracting the thin disk component, the thick disk local population became more representative and showed a progressive approach to axial symmetry, according to decreasing values of the vertex deviation of its velocity ellipsoid.

A more detailed analysis of such a trend towards axial symmetry is needed to improve the estimation of the galactocentric solar radial velocity, since it could be determined by working from an extreme axial and steady state thick disk or halo population. We believe that the more precise determination of such an asymptotic population can answer the question posed by Famaey et al. (2005) about a subset of stars in the solar neighbourhood having no net radial motion, which can be used as a reference against which to measure the solar motion.

This analysis is carried out by using the relationships provided by the point-axial kinematic model, rather than by a linear approximation, enabling us to relate the radial mean velocity and the axiality index, and leading to a more precise extrapolation of the asymptotic behaviour of such an axisymmetric and stationary population of the thick disk in the solar neighbourhood.

In general, the local mean velocity is obtained from an estimation of Oort constants, which obviously are extremely sensible to the working sample, especially if it contains a mixture of differentiated kinematic populations.

On the other hand, the relationship between these constants, the mean velocity, and its gradients are constrained by the symmetry assumptions of the dynamical model. For example, it is well known that there are only two non-null Oort constants, $A$ and $B$, if axial symmetry and pure differential rotation are assumed. Otherwise, if only axial symmetry is assumed, the constant $K$ is generally non-null, but $C$ still vanishes.

In previous work we adopted a quite general framework for the dynamical model under the following assumptions: (1) the stellar system is statistically homogeneous, so that the motion of the stars admits an isolating integral that is quadratic in the peculiar velocities (Chandrasekhar 1942); (2) the fundamental equation of stellar dynamics is satisfied; (3) the hypotheses of a Galactic plane of symmetry and point-to-point axial symmetry (that is, symmetry for opposite and equidistant points through the axis of rotation of the Galaxy) are assumed.

The above assumptions enabled us to consider a stellar system that is quite close to the actual situation. Thus, for example, (a) spiral or barred structures can be described, (b) population parameters are time-dependent, (c) radial and rotation differential movement are allowed, (d) for a fixed time, the orbit of a local population centroid is elliptical, and (e) since the fundamental equation is linear in the phase space density function, the whole stellar system can be described as a mixture of populations.

Therefore, to carry out our analysis, it is necessary to use some consequences of the described model, which are reviewed in the following section. 


\section{Radial and rotation mean velocities}

In the Galactic plane, and according to previous assumptions, the population mean velocity can be expressed by using a galactocentric cylindrical coordinates system, $(r, \theta, z)$ for positions and $(\Pi, \Theta, Z)$ for velocities (with the Galactic rotation defining the positive sign of the angles), as follows (Sanz-Subirana \& Català-Poch 1987; Juan-Zornoza et al. 1990):

$$
\begin{aligned}
& \Pi_{0}=\left[\frac{\dot{k}_{1}}{k_{1}}+\frac{\beta a^{\prime}}{k_{1}^{2}-Q^{2}+k_{2} a r^{2}}\right] \frac{r}{2} \\
& \Theta_{0}=\frac{-\beta a r}{k_{1}^{2}-Q^{2}+k_{2} a r^{2}},
\end{aligned}
$$

where $k_{1}$ and $Q$ are arbitrary functions of time, $\beta$ and $k_{2}$ are constants, and $a$ is a function of time and angle. The time derivative is denoted by a point and the angle derivative by a prima. In particular, for an axial symmetric system we get $Q=0$ and $a=k_{1}$. Integrability conditions for the centroid orbit lead to the following particular function of the angle and time for $a$

$a(\theta, t)=f(\theta) g(t)$.

Under the point-axial model, Oort constants are in general nonnull. Otherwise, under axial symmetry the radial mean velocity becomes

$\Pi_{0}^{(\mathrm{cyl})}=\frac{\dot{k}_{1}}{k_{1}} \frac{r}{2}$,

leading to a vanishing Oort constant $C$. Thus, an axiality index can be defined to quantify the deviation of the stellar population from axial symmetry, according to

$I=\frac{C}{A+B}$

Let us point out two properties of the axiality index. First, $I$ is a function depending only on the angular coordinate, since, by Eq. (2), the following relationship is satisfied

$I(\theta)=\frac{a^{\prime}(\theta, t)}{2 a(\theta, t)}=\frac{f^{\prime}(\theta)}{2 f(\theta)}$.

Second, the axiality index can be expressed explicitly depending on the second central moments, according to the equation

$I(\theta)=-\frac{\mu_{12}}{\mu_{22}}$

(the subindexes 1 and 2 refer to the $\Pi$ - and $\Theta$-velocities, respectively).

Another index may be defined to describe how the stellar velocity distribution differs from a spherical distribution. This is the anisotropy index,

$M=\frac{\mu_{11}}{\mu_{22}}$,

although sometimes it is also defined according to the expression $1-\frac{\mu_{22}}{\mu_{11}}$ (e.g., Binney \& Tremaine 1987).

Notice that, by combining both indexes of Eqs. (6) and (7), the vertex deviation $\delta$ of the velocity ellipsoid is given by

$\tan (2 \delta)=\frac{2 \mu_{12}}{\mu_{11}-\mu_{22}}=\frac{2 I}{I-M}$

(obviously there is some vertex deviation only if the distribution is not spherical, $M \neq 1$ ).
From the study of the mean velocity field (Juan-Zornoza 1995) the mean population velocity in the Galactic plane can be expressed by using Oort constants, together with the foregoing indexes. Thus, taking into account Eq. (3), the equations in Eq. (1) may be written as

$$
\begin{aligned}
& \Pi_{0}=\Pi_{0}^{(\mathrm{cyl})}-\frac{\left(M+I^{2}\right)(B+A)-2(B-A)}{M-I^{2}} I r \\
& \Theta_{0}=\frac{\left(M+I^{2}\right)(B+A)-2(B-A)}{M-I^{2}} r .
\end{aligned}
$$

Therefore, from the above equations we have a relationship basically involving the radial and rotation velocities and the axiality index:

$\Pi_{0}=\Pi_{0}^{(\mathrm{cyl})}-I(\theta) \Theta_{0}\left(I^{2}\right)$.

Let us note that if the foregoing equation is divided by $r$, the term $r^{-1} \Pi_{0}^{(\mathrm{cyl})}$ depends only on the time variate. Thus, from Eq. (3), if the system is in steady state, this term becomes null. Moreover, if the stellar system is axisymmetric, the second term of the radial mean velocity also becomes null.

\section{Nearly axisymmetric and steady state population}

Now we wish to describe the behaviour of the extremely thick disk stars by assuming that: (a) their stellar distribution is close to axisymmetry so that, according to Eq. (6), $I \rightarrow 0$, and (b) their average age is consistent with a nearly vanishing time evolution of their population parameters (Gómez et al. 1997; Soubiran et al. 2003).

If the above conditions are exactly fulfilled we have, by (a), that the second term of the right hand side in Eq. (10) is null, and, by (b) and by taking into account Eq. (3), the first term is also zero. Hence, such a population would have a null radial mean velocity, as is expected for the oldest disk and halo stars (Chiba \& Beers 2000; Vallenari et al. 2006).

Notice that under the described situation, Oort constants satisfy

$A=-\frac{1}{2}\left(\frac{\partial \Theta_{0}^{(\mathrm{cyl})}}{\partial r}-\frac{\Theta_{0}^{(\mathrm{cyl})}}{r}\right), \quad B=-\frac{1}{2}\left(\frac{\partial \Theta_{0}^{(\mathrm{cyl})}}{\partial r}+\frac{\Theta_{0}^{(\mathrm{cyl})}}{r}\right)$,

and also (e.g., Chandrasekhar 1942)

$\frac{B-A}{B}=M$.

Thus, the behaviour of the radial mean velocity around $I=0$, by Eq. (9), is obtained from the following power series of $I$,

$$
\begin{aligned}
\Theta_{0}\left(I^{2}\right) & =\Theta_{0}(0)+\left(\frac{\partial \Theta_{0}\left(I^{2}\right)}{\partial I^{2}}\right)_{I=0} I^{2}+O\left(I^{4}\right) \\
& =(A-B) r+\frac{2 A}{M} r I^{2}+O\left(I^{4}\right) .
\end{aligned}
$$

Taking into account Eq. (11), we alternatively write

$$
\Theta_{0}\left(I^{2}\right)=\Theta_{0}^{(\mathrm{cyl})}-\frac{r^{2}}{M} \frac{\partial}{\partial r}\left(\frac{\Theta_{0}^{(\mathrm{cyl})}}{r}\right) I^{2}+O\left(I^{4}\right) .
$$

In the solar neighbourhood, the first and second terms of the foregoing series are positive. The first one due to the positive sign of the Galactic rotation in the reference frame we are using, and the second one since the derivative is negative according to the signs $\beta<0, k_{1}>0$, and $k_{2}>0$ (Sala 1990). 
Let us remark that the axiality index for the local thick disk population was $|I| \approx 0.2$ (the set of partial thick disk populations that were obtained in Cubarsi \& Alcobé (2004) had decreasing values of $|I|$ from 0.3 to 0.2$)$. Thus, the terms $O\left(I^{4}\right)$ are clearly negligible.

Therefore, by omitting higher order terms, we can eliminate $I$ between Eqs. (14) and (10). Then, the excess of the radial mean velocity of a nearly axial population, with reference to a pure axially symmetric population, can be measured through the following function of the excess of its mean rotation velocity:

$\left(\Pi_{0}-\Pi_{0}^{(\mathrm{cyl})}\right)^{2}=\frac{1}{\rho} \Theta_{0}^{2}\left(\Theta_{0}-\Theta_{0}^{(\mathrm{cyl})}\right)$

with $\rho=-\frac{r^{2}}{M} \frac{\partial}{\partial r}\left(\frac{\Theta_{0}^{(\mathrm{cyl})}}{r}\right)>0$, which is constant for fixed $r$. Hence, such a relationship shows how the trend toward an axially symmetric population, $\Pi_{0} \rightarrow \Pi_{0}^{(\mathrm{cyl})}$ and $\Theta_{0} \rightarrow \Theta_{0}^{(\mathrm{cyl})}$, is constrained in a system close to axisymmetry. In addition, for an asymptotic stationary population $\Pi_{0}^{(\mathrm{cyl})} \rightarrow 0$.

\section{No net radial motion point}

For the actual solar sample, the local thick disk was progressively segregated by working from increasing nested subsamples with the full space motions. The total sample (see details in Cubarsi \& Alcobé 2004) was obtained by crossing the Hipparcos Catalogue (ESA 1997) with radial velocities from the Hipparcos Input Catalogue INCA (ESA 1992). To obtain a representative sample of the Galactic disk, the sample was limited to a trigonometric distance of $300 \mathrm{pc}$. The resulting total sample was composed of 13678 stars, where the only input data were the velocity components $(U, V, W)$ in a Cartesian heliocentric coordinates system, with $U$ toward the Galactic centre, $V$ in the rotational direction, and $W$ perpendicular to the Galactic plane and positive in the direction of the North Galactic pole. The subsamples were obtained according to Alcobé \& Cubarsi (2005) by filtering the whole sample by the absolute value of the total space motion, $145 \leq|\boldsymbol{V}|_{\max } \leq 210 \mathrm{~km} \mathrm{~s}^{-1}$.

According to Eq. (15), the differential radial mean velocity referring to a cylindrical system, $U_{0}-U_{0}^{\text {(cyl) }}$, was plotted against the galactocentric rotation mean velocity $\Theta_{0}$ (Fig. 1). In the new coordinates system, the increment of radial velocity is obtained from Eqs. (6) and (10) as

$\Delta U_{0}=U_{0}-U_{0}^{(\mathrm{cyl})}=\frac{\mu_{U V}}{\mu_{V V}} \Theta_{0}$.

As is shown in Fig. 1, the rotation mean velocity of the axially symmetric and steady state thick disk is extrapolated at $\Delta U_{0}=0$, leading to a value of $\Theta_{0}^{\text {(cyl) }}=138 \pm 2 \mathrm{~km} \mathrm{~s}^{-1}$. Notice that this point satisfies $U_{0}=U_{0}^{(\text {cyl })}=0$.

On the other hand, Fig. 2 displays the location of the population $A_{2}$, which we associated with young disk stars (Alcobé \& Cubarsi 2005), as well as the progressive partial thick disks up to the population $T$ of the complete sample, which are clearly aligned in the plane of radial-rotation heliocentric velocities $U V$.

Let us remark that, in the previous paper, we isolated two non-Gaussian components within the thin disk, which were labeled as $A_{1}$ and $A_{2}$. Both components had the mean velocities and the rotation and vertical velocity dispersions well defined, but the radial velocity dispersion was obtained with a great error, which indicates the non-Gaussianity of these subcomponents. We showed that the subcomponent $A_{2}$ approximately satisfied the asymmetric drift relationship, similarly to older thin

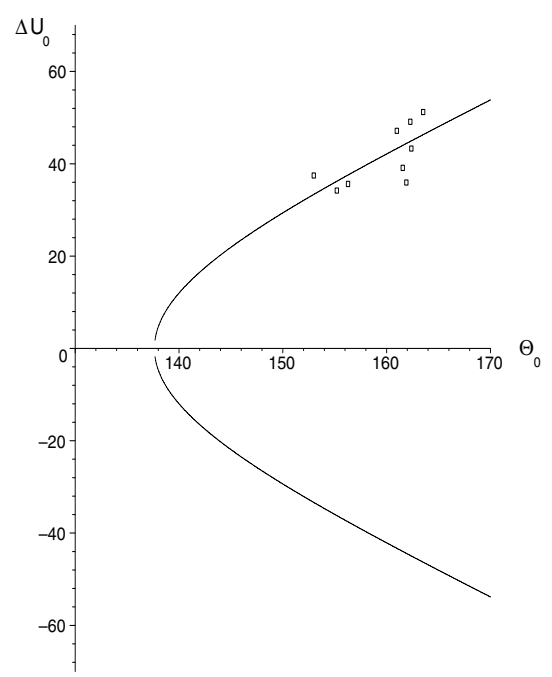

Fig. 1. According to a point-axial system close to axial symmetry, the graph shows how the excess of radial mean velocity $\Delta U_{0}=U_{0}-U_{0}^{\text {(cyl) }}$, referring to a pure axial system, and the mean rotation velocity $\Theta_{0}$ are related. The plotted points correspond to several increasing thick disk populations selected for $145 \leq|\boldsymbol{V}|_{\max } \leq 210 \mathrm{~km} \mathrm{~s}^{-1}$. The continuous line is the regression curve of the points according to Eq. (15), which intersects the horizontal axis at $\Theta_{0}^{(\mathrm{cyl})}=138 \pm 2 \mathrm{~km} \mathrm{~s}^{-1}$. This value corresponds to the mean rotational velocity of the extrapolated thick disk.

disk stars, although neither the subcomponent $A_{1}$ nor the thick disk component fulfilled such a relation. In addition, we compared our segregated populations with the analysis of the thin disk small-scale structure by Soubiran \& Girard (2005). They had found that there was a different chemical and kinematical behaviour between the super metal rich part and the rest of the thin disk. The half of the thin disk stars with $[\mathrm{Fe} / \mathrm{H}]>+0.20$ had motion consistent with that of the Hyades-Pleiades supercluster, which in the velocity space can be associated with the subcomponent $A_{1}$. On the other hand, the other part was composed of older stars, which was consistent with the fact that our subcomponent $A_{2}$ satisfied the typical asymmetric drift relation for disk stars. For this reason, although there is in fact some mixture of stars with different ages in both subcomponents, and for the sake of referring to them according to their kinematic behaviour, we named them early type stars and young disk stars, respectively.

We also showed that, by selecting samples from a maximum heliocentric velocity $|\boldsymbol{V}|_{\max }>125 \mathrm{~km} \mathrm{~s}^{-1}$, a clear discontinuity in the velocity dispersions was found, by detaching thin and thick disks. However, we also found that there was a continuous trend involving the mean velocities of young disk stars, old thin disk stars, and the thick disk population. Such a continuous trend corresponds to the nearly linear fitting that is displayed in Fig. 2.

Thus, that regression line is extrapolated up to the heliocentric rotation velocity of the asymptotic thick disk, $V=-82 \pm$ $2 \mathrm{~km} \mathrm{~s}^{-1}$, with zero radial velocity (by assuming a local mean rotation velocity $\Theta_{\odot}=220 \mathrm{~km} \mathrm{~s}^{-1}$ ). That is, we are able to determine the heliocentric mean radial velocity corresponding to a galactocentric mean rotation velocity $\Theta_{0}^{(\mathrm{cyl})}=138 \mathrm{~km} \mathrm{~s}^{-1}$ for such an asymptotic population.

Hence the heliocentric radial velocity of such an axisymmetric population with no radial movement may be estimated as $U=-20 \pm 1 \mathrm{~km} \mathrm{~s}^{-1}$, referring to the Sun. Such radial velocity is very similar to the mean radial velocity of early-type subcomponent $A_{1}$, which was isolated within the thin disk structure. 


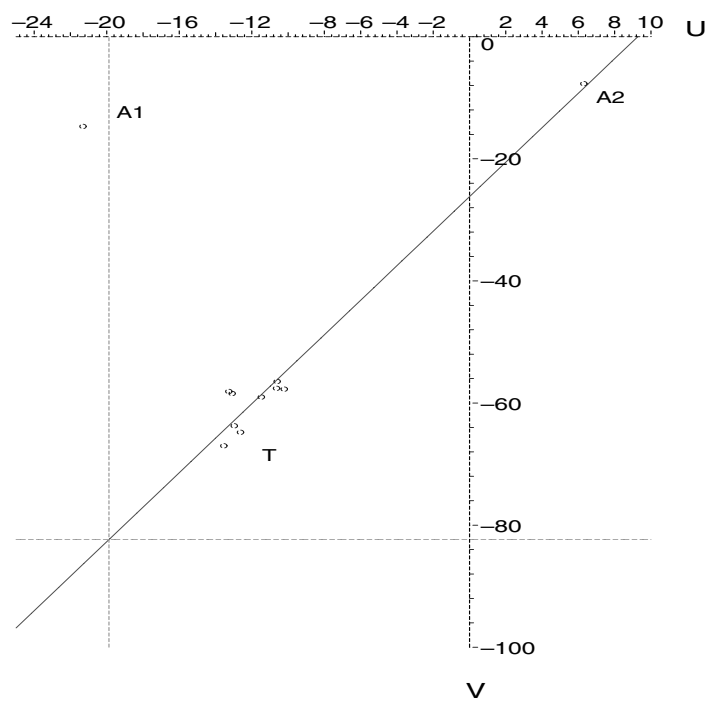

Fig. 2. The graph shows the location of the young disk population centroid $A_{2}$ and partial disk populations up to the complete thick disk $T$, which share a common direction in the plane of radial-rotation heliocentric velocities $U V$. Their straight line fit is extrapolated up to the heliocentric rotation velocity $V=-82 \pm 2 \mathrm{~km} \mathrm{~s}^{-1}$ (horizontal dashed line) of the asymptotic thick disk, with zero radial mean velocity. Hence the heliocentric radial velocity of such an axisymmetric population may be estimated as $U=-20 \pm 1 \mathrm{~km} \mathrm{~s}^{-1}$ (vertical dashed line), which is very close to the mean radial velocity of the early-type population $A_{1}$.

\section{Discussion}

According to Famaey et al. (2005), the subcomponent associated with the Hyades-Pleiades supercluster originated from a common large molecular cloud radially perturbed by a spiral wave. Similarly, Skuljan et al. (1999) described a complex three branch structure between the subcomponents $A_{1}$ and $A_{2}$, along the major axis of the thin disk, which they suggest to be associated with the presence of several adjacent spiral arms. In addition, Soubiran \& Girard (2005) explained the effect of a Galactic bar moving stars from circular to eccentric orbits. The above arguments enable us to give some interpretations of our results.

The non-Gaussianity, in the radial direction, of the velocity distribution of early type stars $A_{1}$ and young disk stars $A_{2}$ is completely consistent with the assumption of a radial perturbation, which would not allow such subcomponents to reach the statistical equilibrium.

The centroid of the $A_{1}$ subcomponent is moving on a nearly circular orbit, $A_{2}$ is moving on an elliptical orbit, and $A_{2}$ has a kinematic behaviour corresponding to stars older than $A_{1}$. Therefore it is plausible to admit that, in the solar neighbourhood and according to Fig. 2, starting from newborn stars, a radial perturbation impels stars toward the Galactic centre up to the stage of the youngest disk population $A_{2}$.

We already mentioned that thin and thick disks were successfully deconvolved, despite having overlapping broad wings, which could produce the effect of an intermediate population. The discontinuity between both disks, which is usually related to how the thick disk was created, could clearly be seen from their velocity dispersions. In the previous paper we showed that before and after the discontinuity, both disk populations had some kind of heating mechanism, but also we found, as mentioned above, that despite the discreteness of the disks, the radial and rotation mean velocities maintained an intriguing nearly constant proportion. Thus we can affirm that, as they get older, the

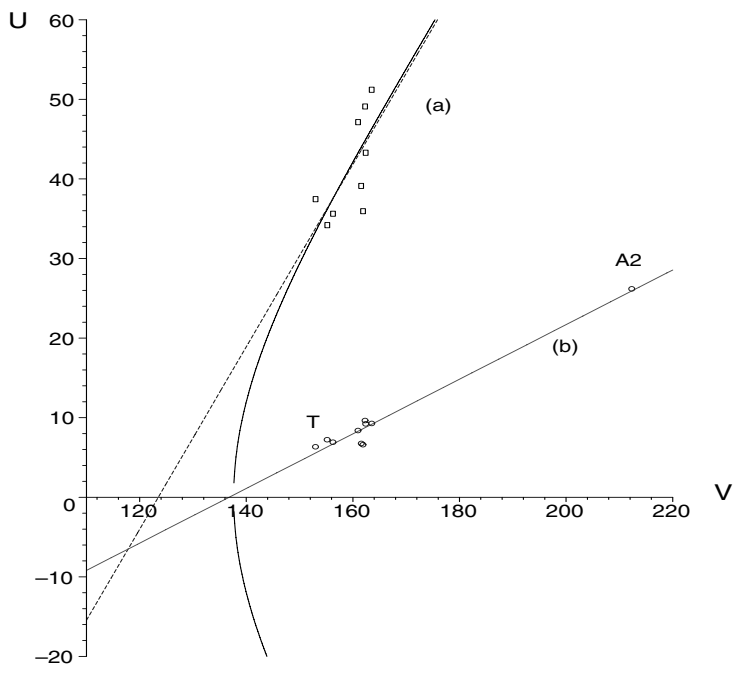

Fig. 3. For increasing thick disk populations, the difference between both regression curves, a) $U_{0}-U_{0}^{(\mathrm{cyl})}$ and b) $U_{0}$, measures the nonvanishing time-dependent term $\Pi_{0}^{(\mathrm{cyl})}$ of the radial mean velocity for a pure axial system. The graphic uses galactocentric $U V$ velocities. The discontinuous line shows the estimation of the no net radial motion point obtained from a linear extrapolation as in previous work.

disk populations partially lose their average angular momentum at the same time that they lose radial motion and increase their velocity dispersion. In addition, the sequences of the oldest thick disk stars follow a trend of steady state and axiality toward the no net radial motion point.

Finally, by putting together previous plots in Fig. 3, now in a galactocentric $U V$ plane, the difference between both regression curves, (a) $U_{0}-U_{0}^{(\mathrm{cyl})}$ and (b) $U_{0}$, allows us to estimate the time dependent term $-U_{0}^{(\mathrm{cyl})}=\Pi_{0}^{(\mathrm{cyl})}$ of Eq. (1), which is clearly non-null for the local thick disk.

We can conclude that both terms contributing to the mean radial velocity in Eq. (10) are acting in opposite senses and are more important for younger than for older stars. The term due to the deviation from axial symmetry increases the radial motion toward the Galactic centre, while the time dependent term opposes it in an attempt to recover the axial symmetry, which is reached for the extrapolated population.

\section{References}

Alcobé, S., \& Cubarsi, R. 2005, A\&A, 442, 929

Binney, J., \& Tremaine, S. 1987, Galactic Dynamics (Princeton: Princeton Univ. Press)

Chandrasekhar, S. 1942, Principles of Stellar Dynamics (New York: Dover Publications Inc.)

Chiba, M., \& Beers, T. C. 2000, AJ, 119, 2843

Cubarsi, R., \& Alcobé, S. 2004, A\&A, 427, 131

ESA 1992, The Hipparcos Input Catalogue, ESA SP-1136

ESA 1997, The Hipparcos Catalogue, ESA SP-1200

Famaey, B., Jorissen, A., Luri, X., et al. 2005, A\&A, 430, 165

Gómez, A. E., Grenier, S., Udry, S., et al. 1997, in Hipparcos- Venice'97, ESA SP-402, 621

Juan-Zornoza, J. M., Sanz-Subirana, J., \& Cubarsi, R. 1990, Ap\&SS, 170, 343 Juan-Zornoza, J. M. 1995, Ph.D. Thesis, Barcelona: Universitat de Barcelona Sala, F. 1990, A\&A, 235, 85

Sanz-Subirana, J., \& Català-Poch, M. A. 1987, in 10th ERAM of the IAU, 4, 267

Skuljan, J., Hearnshaw, J. B., \& Cottrell, P. L. 1999, MNRAS, 308, 731

Soubiran, C., \& Girard, P. 2005, A\&A, 438, 139

Soubiran, C., Bienaymé, O., \& Siebert, A. 2003, A\&A, 398, 141

Vallenari, A., Pasetto, S., Bertelli, G., et al. 2006, A\&A, 451, 125 